\title{
Disfunção Arterial como Possível Mecanismo de Agressão Cardíaca em Pacientes com Diabetes Mellitus Tipo II. Estudo Doppler-Ecocardiográfico
}

\author{
Beatriz Bojikian Matsubara, Katashi O koshi, Marina Politi O koshi, Luiz Shiguero Matsubara, \\ W alkyria P. Pimenta, José C. Christovan, Carlos R. Padovani, Antonio Carlos Cicogna \\ Botucatu, SP
}

Objetivo - Avaliar a estrutura e função do ventrículo esquerdo (VE) e a rigidez arterial em portadores de diabetes mellitus tipo II.

Métodos - Foram estudados 13 doentes diabéticos de ambos os sexos (55 \pm 8 anos) sem outras doenças. A estrutura e função do VE foram avaliadas por meio de ecodopplercardiografia associada à monitorização não invasiva da pressão arterial (PA). Os resultados foram comparados aos obtidos em grupo de indivíduos normais de mesma idade $(n=12)$.

Resultados - Não houve diferenças entre os grupos quanto a PA diastólica, dimensões das câmaras esquerdas e índices de função sistólica e diastólica. Os pacientes diabéticos apresentaram índice de massa do VE $(101 \pm 10 \mathrm{vs}$ $\left.80 \pm 14 \mathrm{~g} / \mathrm{m}^{2} ; p<0,001\right)$ e índice de rigidez arterial sistêmica $(0,86 \pm 0,26$ vs $0,69 \pm 0,19 \mathrm{mmHg} / \mathrm{mL} ; p<0,05)$ significantemente maiores que os controles.

Conclusão - O diabetes mellitus está associado a aumento da rigidez arterial sistêmica e esse fator poderia contribuir para seus efeitos adversos sobre o VE.

Palavras-chave: massa miocárdica, rigidez arterial sistêmica, hipertensão arterial

\section{Arterial Dysfunction as a Possible Mechanism of Cardiac Injury in Type II Diabetic Patients. A Doppler-Echocardiographic Study}

Purpose - To evaluate the left ventricular structure and function and the arterial stiffness in type II diabetic patients.

Methods - Thirteen diabetic patients, men and women (age 55 \pm 8 years) were included in the study. None of the patients had any other clinical disorders. Doppler-echocardiography and non-invasive monitoring of arterial blood pressure were performed. All the results were compared to an age and sex matched control group $(n=12)$.

Results - There were no differences between the groups for diastolic blood pressure, dimensions of cardiac chambers and ventricular systolic and diastolic function indexes. Diabetic patients had increased left ventricular mass index (101 \pm 10 vs $\left.80 \pm 14 \mathrm{~g} / \mathrm{m}^{2} ; \mathrm{p}<0.001\right)$ and increased arterial stiffness $(0.86 \pm 0.26$ vs $0.69 \pm 0.19 \mathrm{mmHg} / \mathrm{mL} ; p<0.05)$, when compared to control group.

Conclusion - Diabetes mellitus is associated to increased systemic arterial stiffness and this may contribute to the adverse effects of diabetes on left ventricular morphology.

Key-words: myocardial mass, systemic arterial stiffness, arterial hypertension

Arq Bras Cardiol, volume 69 (n 3), 155-159, 1997

Estudos realizados em situações experimentais e clínicas demonstraram que o diabetes mellitus inclui anormalidades metabólicas crônicas ${ }^{1,2}$ que induzem alterações estruturais ${ }^{3-7}$ e funcionais ${ }^{5,8-10}$ do miocárdio, mesmo na au-

Faculdade de Medicina de Botucatu - UNESP. Apoio FAPESP - Proc 91/3666-9 Correspondência: Beatriz Bojikian Matsubara - Depto de Clínica Médica Faculdade de Medicina de Botucatu-UNESP - 18618-000 - Botucatu, SP Recebido para publicação em 3/4/97

Aceito em 23/7/97 sência de outros fatores de risco para cardiopatias, tais como hipertensão arterial (HA) e doença aterosclerótica coronária. Estes achados levaram vários autores ${ }^{1-4,8,9}$ a sugerir a existência de doença intrínseca do miocárdio nos portadores de diabetes mellitus.

Entretanto, ainda é controversa a existência de uma cardiomiopatia específica associada a esta doença ${ }^{11,12}$. Borow e col ${ }^{12}$ estudaram a função miocárdica de adultos jovens portadores de diabetes mellitus tipo I, utilizando ín- 
dices de contratilidade não sujeitos a influências das condições de carga. Verificaram que, nessa população normotensa, sem insuficiência coronária ou microangiopatia diabética, a contratilidade miocárdica era normal. Os autores concluíram que, embora seja comum a presença de doença cardíaca em pacientes diabéticos, a elevada morbidade e a mortalidade cardíacas não podem ser associadas a alterações intrínsecas da contratilidade miocárdica.

Outro fator que pode estar envolvido na gênese da doença cardíaca em diabéticos é a presença de alterações vasculares periféricas nestes doentes. Trabalhos recentes ${ }^{13-16}$ demonstraram modificações das propriedades mecânicas de grandes artérias em portadores de diabetes mellitus ou, tão somente, apresentando teste de tolerância à glicose anormal ${ }^{16}$. Essas evidências podem ampliar o espectro dos possíveis mecanismos fisiopatológicos envolvidos no comprometimento miocárdico associado ao diabetes mellitus.

O objetivo do presente estudo foi analisar, por meio de método não invasivo, a morfologia e a função do ventrículo esquerdo (VE) de pacientes normotensos portadores de diabetes mellitus não insulino-dependente (DMNID), sem evidências de insuficiência coronária ou microangiopatia e avaliar a rigidez arterial sistêmica destes pacientes.

\section{Métodos}

Pacientes normotensos (pressão arterial sistólica (PAS) <140mmHg e pressão arterial diastólica (PAD) $<90 \mathrm{mmHg}^{17}$ ), portadores de DMNID foram triados nos ambulatórios dos Postos de Saúde municipais de Botucatu e de Clínica Médica Geral e Endocrinologia do Hospital das Clínicas da Faculdade de Medicina de Botucatu. Indivíduos voluntários normais foram selecionados para compor o grupo controle.

A partir da triagem inicial, os doentes foram submetidos a minuciosa avaliação clínica e laboratorial. Os pacientes diabéticos eram controlados apenas com dieta ou com uso de hipoglicemiantes orais ou insulina. $\mathrm{O}$ grupo diabetes foi composto apenas pelos casos que obedeciam aos critérios de seleção: 1) ausência de antecedentes de etilismo ou tabagismo; 2) ausência de sintomas sugestivos de isquemia miocárdica ou insuficiência cardíaca; 3) exame clínico do coração normal; 4) eletrocardiograma e radiografia de tórax normais; 5) glicemia de jejum <140mg/dL; 6) glicosúria $<5,0 \mathrm{~g}$ em urina de $24 \mathrm{~h}$; 7) exame de fundo de olho normal; 8) proteinúria de $24 \mathrm{~h}<300 \mathrm{mg}$. Os critérios 5 e 6 foram utilizados para definir o controle do diabetes como sendo bom e aceitável ${ }^{18}$ e os critérios 7 e 8 para excluir a presença de microangiopatia diabética.

Os voluntários normais foram submetidos às mesmas avaliações. Doze indivíduos preencheram todos os critérios de inclusão e foram admitidos no grupo controle $(n=12)$.

Todos os parâmetros de morfologia e função cardíacas e de rigidez arterial sistêmica foram obtidos por meio de Doppler-ecocardiografia associada a monitorização não invasiva da pressão arterial (PA) sistêmica.
Os exames foram realizados no período da manhã, entre 8:00 e 11:00h, estando os pacientes e os indivíduos voluntários normais alimentados, e após período de repouso de 15 min em ambiente calmo e com temperatura controlada.

Os ecocardiogramas (ECO) foram obtidos utilizandose o equipamento Ultramark 8 (Advanced Technology Laboratories), com transdutor de 3,0MHz. A PA sistêmica foi aferida por meio de esfigmomanômetro com coluna de mercúrio, no braço esquerdo, com o paciente na mesma posição em que o ECO foi realizado. Três medidas foram obtidas, tomando-se a média aritmética de seus valores como referência.

As medidas ecocardiográficas foram efetuadas seguindo-se as recomendações da American Society of Echocardiography ${ }^{19}$. Os seguintes índices foram calculados utilizando-se as medidas monodimensionais do VE: 1) fração de ejeção $(\mathrm{FE})=\left[\left(\mathrm{DDVE}^{3}-\mathrm{DSVE}^{3}\right) / \mathrm{DDVE}^{3}\right]$, onde DDVE e DSVE correspondem aos diâmetros diastólico e sistólico do VE; 2) porcentagem de variação do diâmetro ventricular $(\% \Delta \mathrm{D}): \% \Delta \mathrm{D}=[(\mathrm{DDVE}-\mathrm{DSVE}) / \mathrm{DDVE}] \times 100$; 3) espessura relativa da parede $(\mathrm{H} / \mathrm{R}): \mathrm{H} / \mathrm{R}=[(\mathrm{SIVd}+\mathrm{PPVEd}) /$ 2]/DDVE, onde SIVd e PPVEd correspondem às espessuras diastólicas do septo interventricular e da parede posterior do VE; 4) massa do VE $(\mathrm{MVE})^{20}: \mathrm{MVE}(\mathrm{g})=\{[(\mathrm{SIVd}+\mathrm{PPVEd}$ +DDVE) ${ }^{3}$-DDVE $\left.\left.{ }^{3}\right] \mathrm{x} 1,04\right\}-13,6$. O valor de MVEfoi normalizado para superfície corporal, constituindo o índice de massa do VE (IMVE, g/m²); 5) stress sistólico final (SSF) ${ }^{(21)}$ : SSF $\left(\mathrm{g} / \mathrm{cm}^{2}\right)=1,36 \times(\mathrm{PAS} \times \mathrm{DDVE}) /[4 \mathrm{Hs}(1+\mathrm{Hs} / \mathrm{DDVE})]$, onde PAS é a pressão arterial sistólica e Hs é a espessura sistólica média da parede ventricular, correspondente à média dos valores das espessuras sistólicas do septo interventricular e da parede posterior do VE.

As características do fluxo diastólico através da valva mitral e do fluxo sistólico através da valva aórtica foram analisadas por meio de Doppler pulsado. No $1^{\circ}$ caso, posicionou-se a amostra na via de entrada do VE, na borda da valva mitral, visibilizada na posição padrão de quatro câmaras. No caso da valva aórtica, a amostra foi posicionada na via de saída do VE, logo abaixo dos folhetos aórticos.

Os seguintes índices de função sistólica e diastólica foram calculados: 1) razão entre os picos de velocidade diastólica inicial e final através da valva mitral (E/A);2) tempo de relaxamento isovolumétrico (TRI, ms), equivalente ao tempo decorrido entre o final do fluxo sistólico pela valva aórtica e o início do fluxo diastólico pela valva mitral; 3) tempo de desaceleração do fluxo diastólico inicial através da valva mitral (DesE, cm/s²); 4) volume sistólico (VS, mL) foi calculado utilizando-se a integral do fluxo sistólico e o diâmetro sistólico da via de saída do VE; 5) índice cardíaco (IC, $\left.\mathrm{L} / \mathrm{min} / \mathrm{m}^{2}\right)=(\mathrm{FCxVS}) / \mathrm{SC}$, onde FC é a frequiência cardíaca e $\mathrm{SC}$ é superfície corporal; 6$)$ índice de rigidez arterial $(\Delta \mathrm{P} / \Delta \mathrm{V}$, $\mathrm{mmHg} / \mathrm{mL})=$ pulso de $\mathrm{PA} /$ volume sistólico ${ }^{13}$. O pulso de $\mathrm{PA}$ corresponde à diferença entre as PAS e PAD, aferidas pelo esfignomanômetro e o volume sistólico corresponde ao valor do parâmetro descrito no item 4 . Como resultado, obteve-se a variação de PA para cada $\mathrm{mL}$ de sangue ejetado na aorta. Quanto maior o valor do índice, mais rígidoé o 


\begin{tabular}{|lccccc|}
\hline \multicolumn{5}{|c|}{$\begin{array}{c}\text { Tabela I - Valores médios e respectivos desvios padrão de parâmetros } \\
\text { clínicos de pacientes diabéticos (grupo diabetes) e de indivíduos } \\
\text { normais (grupo controle) }\end{array}$} \\
\hline Grupo & $\begin{array}{l}\text { Idade } \\
(\text { anos })\end{array}$ & $\begin{array}{c}\text { SC } \\
\left(\mathrm{m}^{2}\right)\end{array}$ & $\begin{array}{c}\text { PAS } \\
(\mathrm{mmHg})\end{array}$ & $\begin{array}{c}\text { PAD } \\
(\mathrm{mmHg})\end{array}$ & $\begin{array}{c}\text { FC } \\
(\mathrm{bpm})\end{array}$ \\
\hline $\begin{array}{l}\text { Controle } \\
(\mathrm{n}=12)\end{array}$ & $55 \pm 9$ & $1,83 \pm 0,18$ & $119 \pm 17$ & $77 \pm 8$ & $61 \pm 12$ \\
$\begin{array}{l}\text { Diabetes } \\
(\mathrm{n}=13)\end{array}$ & $55 \pm 8$ & $1,78 \pm 0,20$ & $128 \pm 12 *$ & $81 \pm 7$ & $70 \pm 11$ \\
\hline $\begin{array}{l}\text { SC- superfície corporal; PAS e PAD- pressão arterial sistólica e diastólica; } \\
\text { FC- frequiência cardíaca; } \text { *p }<0,05 \text { vs controle. }\end{array}$ \\
\hline
\end{tabular}

\begin{tabular}{|c|c|c|c|c|c|c|c|}
\hline \multicolumn{8}{|c|}{$\begin{array}{c}\text { Tabela II -Valores médios e desvios padrão das dimensões cardíacas } \\
\text { avaliadas pelo ecocardiograma em pacientes diabéticos (grupo diabetes) } \\
\text { e em indivíduos normais (grupo controle) }\end{array}$} \\
\hline Grupo & $\begin{array}{c}\text { DDVE } \\
(\mathrm{mm})\end{array}$ & $\begin{array}{c}\text { DSVE } \\
(\mathrm{mm})\end{array}$ & $\mathrm{Hd}$ & $\mathrm{H} / \mathrm{D}$ & $\begin{array}{c}\mathrm{AE} \\
(\mathrm{mm})\end{array}$ & $\begin{array}{l}\text { Ao } \\
(\mathrm{mm})\end{array}$ & $\begin{array}{l}\text { IMVE } \\
\left(\mathrm{g} / \mathrm{m}^{2}\right)\end{array}$ \\
\hline $\begin{array}{l}\text { Controle } \\
(\mathrm{n}=12)\end{array}$ & $47 \pm 5$ & $29 \pm 4$ & $8,3 \pm 1,1$ & $0,35 \pm 0,06$ & $37 \pm 4$ & $33 \pm 4$ & $80 \pm 14$ \\
\hline $\begin{array}{l}\text { Diabetes } \\
(\mathrm{n}=13)\end{array}$ & $50 \pm 5$ & $30 \pm 6$ & $8,9 \pm 0,8$ & $0,37 \pm 0,05$ & $39 \pm 4$ & $32 \pm 3$ & $101 \pm 10^{*}$ \\
\hline
\end{tabular}

sistema arterial. Os índices 1 a 3 são relativos à função diastólica e os índices 4 e 5 à função sistólica do VE.

As diferenças entre os grupos foram analisadas por meio do teste $t$ de Student, considerando-se o nível de significância $<0,05$.

\section{Resultados}

Treze pacientes ( $55 \pm 8$ anos) foram admitidos no grupo diabetes e comparados com o grupo controle (55 \pm 9 anos). Na tabela I são apresentados parâmetros clínicos dos dois grupos estudados. Não foram observadas diferenças significativas quanto à idade e superfície corporal. Os pacientes diabéticos, embora normotensos segundo os critérios estabelecidos, apresentaram PAS significantemente maior do que os indivíduos normais. A PAD e a freqüência cardíaca foram comparáveis nos dois grupos.

Os valores das dimensões cardíacas obtidas por meio do ECO convencional estão na tabela II. Não foram observadas diferenças significantes entre os grupos, exceto pelo índice de massa do VE, significantemente maior no grupo diabetes.

Nas tabelas III e IV são apresentados os índices de função sistólica e diastólica do VE e o índice de rigidez arterial sistêmica. Os grupos foram indistinguíveis entre si quanto aos parâmetros de função ventricular; porém, observou-se rigidez arterial sistêmica aumentada no grupo diabetes.

\begin{tabular}{|c|c|c|c|c|c|}
\hline \multicolumn{6}{|c|}{$\begin{array}{l}\text { Tabela III - Valores médios e respectivos desvios padrão de parâmetros } \\
\text { funcionais sistólicos do ventrículo esquerdo de pacientes diabéticos } \\
\text { (grupo diabetes) e de indivíduos normais (grupo controle) }\end{array}$} \\
\hline Grupo & $\mathrm{FE}$ & $\begin{array}{c}\% \Delta \mathrm{D} \\
(\%)\end{array}$ & $\begin{array}{l}\text { VS } \\
(\mathrm{mL})\end{array}$ & $\begin{array}{c}\mathrm{IC} \\
\left(\mathrm{L} / \mathrm{min} / \mathrm{m}^{2}\right)\end{array}$ & $\begin{array}{c}\mathrm{SSF} \\
\left(\mathrm{g} / \mathrm{cm}^{2}\right)\end{array}$ \\
\hline $\begin{array}{l}\text { Controle } \\
(\mathrm{n}=12)\end{array}$ & $0,77 \pm 0,05$ & $39 \pm 5$ & $61 \pm 12$ & $2,2 \pm 0,3$ & $199 \pm 41$ \\
\hline $\begin{array}{l}\text { Diabetes } \\
(\mathrm{n}=13)\end{array}$ & $0,77 \pm 0,08$ & $40 \pm 7$ & $59 \pm 11$ & $2,3 \pm 0,3$ & $207 \pm 35$ \\
\hline
\end{tabular}

FE- fração de ejeção; \% $\Delta \mathrm{D}$ - porcentagem de variação do diâmetro ventricular; VS- volume sistólico; IC- índice cardíaco; SSF- esforço sistólico final (póscarga).

\begin{tabular}{|c|c|c|c|c|}
\hline \multicolumn{5}{|c|}{$\begin{array}{l}\text { Tabela IV - Parâmetros de função diastólica do ventrículo esquerdo e } \\
\text { índice de rigidez arterial }(\Delta \mathrm{P} / \Delta \mathrm{V}) \text { de pacientes diabéticos (grupo } \\
\text { diabetes) e indivíduos normais (grupo controle). Os valores } \\
\text { representam as médias e seus respectivos desvios padrão }\end{array}$} \\
\hline Grupo & $\mathrm{E} / \mathrm{A}$ & $\begin{array}{l}\text { TRI } \\
(\mathrm{ms})\end{array}$ & $\begin{array}{l}\text { Des. E } \\
\left(\mathrm{cm} / \mathrm{s}^{2}\right)\end{array}$ & $\begin{array}{c}\Delta \mathrm{P} / \Delta \mathrm{V} \\
(\mathrm{mmHg} / \mathrm{mL})\end{array}$ \\
\hline $\begin{array}{l}\text { Controle } \\
(\mathrm{n}=12)\end{array}$ & $1,2 \pm 0,3$ & $99 \pm 19$ & $309 \pm 84$ & $0,69 \pm 0,19$ \\
\hline $\begin{array}{l}\text { Diabetes } \\
(\mathrm{n}=13)\end{array}$ & $1,1 \pm 0,4$ & $96 \pm 16$ & $338 \pm 88$ & $0,86 \pm 0,26^{*}$ \\
\hline \multicolumn{5}{|c|}{$\begin{array}{l}\text { E/A- razão entre os picos de velocidade diastólica precoce (E) e tardia (A) } \\
\text { na valva mitral; TRI-tempo de relaxamento isovolumétrico; Des. E-tempo de } \\
\text { desaceleração do fluxo diastólico inicial pela valva mitral; } \Delta \mathrm{P} / \Delta \mathrm{V} \text { - relação } \\
\text { entre pulso de pressão arterial e volume ejetado na aorta (índice de rigidez } \\
\text { arterial); * }<00,05 \text { vs controle. }\end{array}$} \\
\hline
\end{tabular}

\section{Discussão}

A elevada morbidade e mortalidade de causa cardíaca é bem conhecida nos portadores de diabetes mellitus ${ }^{22-24}$. Esse fato levou diversos autores a considerarem a possibilidade da doença envolver alterações estruturais do miocárdio que pudessem levar ao desenvolvimento de insuficiência cardíaca ${ }^{1,2,4,8,9,25,26}$. Tais alterações, segundo essa hipótese, comporiam um quadro de cardiomiopatia correspondente a uma entidade nosológica distinta e especificamente associada ao diabetes ${ }^{8,9,25,26}$.

Enquanto trabalhos experimentais apontam para tal possibilidade ${ }^{8,9,27,28}$, os resultados de investigações clínicas continuam controversos. Alguns autores ${ }^{1-4,25,26}$ defendem a existência da cardiomiopatia diabética, enquanto que outros falharam em demonstrar alterações funcionais cardíacas em pacientes diabéticos normotensos e livres de insuficiência coronária ${ }^{11,12}$. As discrepâncias entre os resultados dos diferentes estudos são devidas, em grande parte, à heterogeneidade das populações avaliadas.

Nossos critérios de seleção dos pacientes foram bastante rigorosos e tal medida, se por um lado comprometeu o número da amostra, por outro lado tornou o grupo estudado bastante homogêneo. Nessa população, os resultados obtidos indicaram que no DMNID, estando ausentes HA, insuficiência coronária e microangiopatia diabética, a função ventricular, sistólica e diastólica, está preservada. Por outro lado, foi interessante constatar que esses pacientes, 
normotensos, apresentaram aumento da rigidez arterial sistêmica, da PAS e do índice de massa ventricular, em relação ao grupo controle.

Trabalhos publicados recentemente ${ }^{13,15,16}$ já relataram aumento da rigidez de grandes artérias em doentes com diabetes mellitus tipo I, tipo II e mesmo em pacientes apresentando apenas o teste de tolerância à glicose alterado ${ }^{16}$. Esses achados, concordantes com nossos resultados, indicariam um mecanismo fisiopatológico adicional de agressão cardíaca que envolveria o sistema arterial periférico como alteração primária.

Salomaa e col ${ }^{16}$ estudaram o comportamento mecânico da artéria carótida comum de pacientes diabéticos e verificaram aumento da rigidez da parede do vaso e diminuição de sua complacência. Os autores discutem que, nesses pacientes, a HA sistólica seria conseqüência de alteração vascular e não causa desta alteração, deixando de analisar as repercussões cardíacas dependentes do sistema arterial mais rígido.

As causas para o comportamento mecânico arterial anormal podem estar relacionadas com modificações estruturais ou funcionais da parede dos vasos. Do ponto de vista anatômico, é referido que nos pacientes diabéticos existiriam depósitos de lípides e outras substâncias na parede das artérias predispondo ao desenvolvimento de aterosclerose e, portanto, modificando as características físicas dos vasos ${ }^{29}$. No entanto, Salomaa e col ${ }^{16}$ observaram, em indivíduos diabéticos ou com tolerância à glicose alterada, que a rigidez do sistema arterial poderia estar aumentada, independentemente da espessura da parede arterial, sugerindo alteração funcional. Sob esse aspecto, existem várias evidências de associação entre hiperglicemia e vasoconstrição arterial $^{30,31}$

Estudos realizados em seres humanos ${ }^{32}$ e em situa- ções experimentais ${ }^{30,33}$ indicaram que a vasodilatação estimulada por drogas está significantemente comprometida em situações de hiperglicemia. Esta perda de função foi associada à produção deficiente de óxido nítrico, que é um fator de relaxamento endotelial ${ }^{34}$.

No presente estudo, os pacientes apresentaram aumento significante do índice de massa miocárdica. Outros autores documentaram achados semelhantes ${ }^{6,35,36}$. Grossman e col ${ }^{37}$ estudaram pacientes hipertensos diabéticos e não diabéticos e concluíram que a doença metabólica acelera a hipertrofia do VEem pacientes com HA, independentemente dos níveis pressóricos. As causas da associação entre diabetes e hipertrofia miocárdica não estão definidas.

Nossos dados, somados aos relatos da literatura, admitem a hipótese de que o aumento da massa miocárdica ocorre em resposta à rigidez anormal do sistema arterial, que é responsável pelo aumento da pós-carga. O crescimento miocárdico permite a normalização da pós-carga e, conseqüentemente, a manutenção da função ventricular normal. Em nosso estudo, embora a PAS fosse significantemente maior nos pacientes diabéticos, o esforço sistólico final, isto é, a pós-carga, foi comparável à do grupo controle.

Concluindo, existe aumento da rigidez arterial sistêmica associado ao diabetes mellitus tipo II, mesmona ausência deHA ou microangiopatia. Estaalteração do comportamentomecânico arterial está associada ao aumento de massa miocárdica e pode constituir mecanismo adicional de morbidade cardíaca que freqüentemente acompanha o diabetes.

\section{Agradecimentos}

Aos Srs. Mário Augusto Dallaqua, Valéria Maria Ricarelli de Oliveira e Renato Borges Pereira pela colaboração prestada.

\section{Referências}

1. Rodrigues B, McNeill JH - The diabetic heart: metabolic causes for the development of a cardiomyopathy. Cardiovasc Res 1992; 26: 913-22.

2. Rodrigues B, Cam MC, McNeill JH - Myocardial substrate metabolism: implications for diabetic cardiomyopathy. J Mol Cell Cardiol 1995; 27: 169-79.

3. Argüelles E, Silva HB, Weksler C - Aspectos clínicos da miocardiopatia diabética. Arq Bras Cardiol 1983; 40: 9-13.

4. Galderisi M, Anderson KM, Wilson PWF, Levy D - Echocardiographic evidence for the existence of a distinct diabetic cardiomyopathy (the Framingham Heart Study). Am J Cardiol 1991; 68: 85-9.

5. Vanninen E, Mustonen J, Vainio P, Lansimies E, Uusitupa M - Left ventricular function and dimensions in newly diagnosed non-insulin-dependent diabetes mellitus. Am J Cardiol 1992; 70: 371-8.

6. Gambardella S, Frontoni S, Spallone V et al - Increased left ventricular mass in normotensive diabetic patients with autonomic neuropathy. Am J Hypertens 1993; 6: 97- 102.

7. Ohya Y, Abe I, Fujii K et al - Hyperinsulinemia and left ventricular geometry in a work-site population in Japan. Hypertension 1996; 27: 729-34.

8. Fein FS, Zola BE, Malhotra A et al - Hypertensive-diabetic cardiomyopathy in rats. Am J Physiol 1990; 258: H793-805.
9. Fein FS, Sonnenblick EH - Diabetic cardiomyopathy. Cardiovasc Drugs Ther 1994; 8: 65-73.

10. Celentano A, Vaccaro O, Tammaro Pet al - Early abnormalities of cardiac function in non-insulin-dependent diabetes mellitus and impaired glucose tolerance. Am J Cardiol 1995; 76:1173-6.

11. Factor SM, Minase T, Sonnenblick EH - Clinical and morphological features of human hypertensive-diabetic cardiomyopathy. Am Heart J 1980; 99: 446-58.

12. Borow KM, Jaspan JB, Willians KA, Neumann A, Wolinski-Walley P, Lang RM - Myocardial mechanics in young adult patients with diabetes mellitus: effects of altered load, inotropic state and dynamic exercise. J Am Coll Cardiol 1990; 15 : 1508-17.

13. Devereux RB, Howard BV, Roman MJ et al - Relation of diabetes and impaired glucose tolerance to left ventricular structure and function in Arizona Indians: the strong heart study. Circulation 1995; 92(suppl I): 419.

14. Kool MJ, Lambert J, Stehouwer CD, Hoeks AP, Boudier HAS, Van Bortel LM Vessel wall properties of large arteries in uncomplicated IDDM. Diabetes Care 1995; 18:618-24.

15. Rydén ÅA, Länne T, Wollmer P, Sonesson B, Hansen F, Sundkvist G - Increased 
arterial stiffness in women, but not in men, with IDDM. Diabetologia 1995; 38:1082-9.

16. Salomaa V, Riley W, Kark JD, Nardo C, Folsom AR - Non-insulin-dependent diabetes mellitus and fasting glucose and insulin concentrations are asssociated with arterial stiffness indexes. The ARIC study. Circulation 1995; 91: 1432-43.

17. Joint National Committee on Detection, Evaluation, and Treatment of High Blood Pressure. The fifth report of the Joint National Committee on Detection, Evaluation, and Treatment of High Blood Pressure (JNC V). Arch Intern Med 1993; 153: 154-83

18. American Diabetes Association - Standards of Medical Care for patients with diabetes mellitus. Diabetes Care 1994; 17: 616-23.

19. Sahn DJ, DeMaria A, Kisseo J, Weyman A - Recommendations regarding quantitation in M-mode echocardiography: results of a survey of echocardiographic measurements. Circulation 1978; 58: 1072-83.

20. Devereux RB, Reichek N - Echocardiographic determination of left ventricular mass in men: anatomic validation of the method. Circulation 1977; 55: 613-8.

21. Reichek M, Wilson J, St John Sutton M, Plappert TA, Goldberg S, Hirshfeld JW - Noninvasive determination of left ventricular end systolic stress: validation of the method and initial application. Circulation 1982; 65: 99-109.

22. Garcia MJ, Mc Namara PM, Gordon T, Kannel WB - Morbidity and mortality in diabetics in the Framingham population sixteen year follow-up study. Diabetes 1974; 23: 105-11.

23. Kannel WP, McGee DL - Diabetes and cardiovascular disease. The Framingham Heart Study. J Am Med Assoc 1979; 241: 2035-8.

24. Curb JD, Rodriguez BL, Burchfiel CM, Abbott RD, Chiu D, Yano K - Sudden death, impaired glucose tolerance, and diabetes in Japanese American men. Circulation 1995; 91: 2591-5.

25. Hamby RI, Zoneraich S, Sherman L - Diabetic cardiomyopathy. J Am Med Assoc 1974; $229: 1749-54$
26. Regan TJ, Lyons MM, Ahmed SS et al - Evidence for cardiomyopathy in familial diabetes mellitus. J Clin Invest 1977; 60: 885-99.

27. Regan TJ,Ettinger PO, Khan MI et al-Altered myocardial function and metabolism in chronic diabetes mellitus without ischemia in dogs. Circ Res 1974; 35: 222-37.

28. Garvey WG, Hardin D, Juhaszova M, Dominguez JH-Effects of diabetes on myocardial glucose transport system in rats: implication for diabetic cardiomyopathy. Am J Physiol 1993; 264: 837-44

29. Sniderman A, Michel C, Racine N-Heart disease in patients with diabetes mellitus. J Clin Epidemiol 1992; 45: 1357-70.

30. Tesfamariam B, Brown ML, Deykin D, Cohen RA - Elevated glucose promote generation of endothelium-derived vasoconstrictor prostanoids in rabbit aorta J Clin Invest 1990; 85: 929-32.

31. Cohen RA - Dysfunction of vascular endothelium in diabetes mellitus. Circulation 1993; 87(suppl V): 67-76.

32. Quyyumi AA, Dakak N, Andrews NP, Gilligan DM, Panza JA, Cannon RO Contribution of nitric oxide to metabolic coronary vasodilation in human heart Circulation 1995; 92: 320-6.

33. Mayhan W, Simmons LK, Sharpe QM-Mechanism of impaired responses of cerebral arterioles during diabetes mellitus. Am J Physiol 1991; 260: H319-26.

34. Mombouli JV, Vanhoutte PM - Kinins and endothelial control of vascular smooth muscle. Annual Rev Pharmacol Toxicol 1995; 35: 679-705.

35. Sharp SD, Williams RR - Fasting insulin and left ventricular mass in hypertensive and normotensive controls. Cardiology 1992; 81: 207-12.

36. Sasson Z, Rasooly Y, Bhesania T, Rasooly I - Insulin resistance is an important determinant of left ventricular mass in the obese. Circulation 1993; 88 1431-6.

37. Grossman E, Shemesh J, Shamiss A, Thaler M, Carroll J, Rosenthal T - Lef ventricular mass in diabetes-hypertension. Arch Intern Med. 1992; 152: 1001-4. 